\title{
Metabolic and Behavioral Patterns in a Pre-Menstrual Syndrome Animal Model with Liver-qi Invasion and Their Reversal by a Chinese Traditional Formula
}

\author{
Peng Sun, Sheng Wei, Huiyun Zhang, Mingqi Qiao* \\ Lab of TCM Classical Theory, MOE, Shandong University of Traditional \\ Chinese Medicine, Jinan, China \\ E-mail: times.wei@gmail.com,qmingqi@163.com \\ Received December 8, 2010; revised December 10, 2010; accepted December 14, 2010
}

\begin{abstract}
This work successfully used model rats with Pre-Menstrual Syndrome (PMS) liver-qi invasion in the early development days to detect the Metabolic and Behavioral Patterns and their reversal by a Chinese traditional formula. Our aim is to verify the reliability of PMS liver-qi invasion rat model and explore some micro- mechanism of the syndrome of the liver failing to maintain the normal flow of qi. 30 rats with estrous cycles not in accepting time were selected and divided randomly into three groups: the normal control group, PMS liver-qi invasion model group and PMS liver-qi invasion medication-administered group. Emotional stimulation and multiple factors combination were used to prepare the PMS liver-qi invasion model. Baixiangdan Capsules (a Chinese traditional formula) were administered to rats to interfere with the PMS liver-qi invasion mode. Open-field test was used to explore behavioral aspects of the model. Urine samples, from the three groups, were collected and analyzed with UPLC-Q-TOF method to detect changes in metabolites related to liver functions. In the open-field experiment, the crossing scores, rearing scores and open-field experiment total scores of rats in the PMS liver-qi invasion model group increased remarkably $(\mathrm{P}<0.05)$ compared with the scores of the normal control group, the tendency was retrieved remarkably after medications $(\mathrm{P}<0.05)$. Metabolic fingerprints between the PMS liver-qi invasion model group and the normal control group had also distinguished changes through principal component analysis, and an evident restoration trend occurred after Baixiangdan Capsules administration. Taken together, behavioral and metabolic patterns can differentiate the PMS liver-qi invasion rat models from the normal rats. Our results identified potential biological markers that might reflect metabolic pathologies associated with PMS liver-qi invasion.
\end{abstract}

Keywords: Premenstrual Syndrome, Liver-Qi Invasion, Open-Field, Metabonomics, Baixiangdan Capsules

\section{Introduction}

Pre-menstrual Syndrome (PMS) is the syndrome which regularly happens before emmenia, and affects women's daily life and work [1]. Animal models of PMS are valuable tools to investigate behavioral, physiological, and molecular aspects associated with PMS [2,3]. From the Chinese Traditional Medical point of view, PMS is a result from the failure of the liver to maintain the normal flow of qi. Its pathogenesis refers to three systems the mind, nerves and internal hormones. The involvement of liver qi is supported by studies on animal models of PMS.

\footnotetext{
*Sun Peng and Wei Sheng contribute equally to the work.
}

For example, increase in anxiety behavior, that is similar to liver-qi invasion induced anxiety, was reported in an animal model of PMS [3]. Although genetic and proteomics can reveal the pathophysiology in a great extent, they also have the defects in the interaction of protein signal pathway and the difficult to locate target sites, and can't dynamically, real-timely reflect the overall information. The present study provides new insights into the pathogenesis of PMS from traditional Chinese medical view. We investigated behavioral characteristics and the metabolic markers in urine in premenstrual syndrome liver-qi invasion rat model. Furthermore, we investigated the potential effects of a traditional Chinese formula (Baixiangdan Capsules) on reversing PMS associated 
behavioral and metabolic changes.

\section{Methods and Materials}

\subsection{Sample Animals}

Thirty healthy Wistar female rats were chosen with body weights from $180 \mathrm{~g}$ to $220 \mathrm{~g}$. They were provided by Experimental Animal Centre of Shandong University of Traditional Chinese Medicine where they initially came from Chinese Rodents Experimental Animal Seed Bank Shanghai Center with production license: SCXK (Shandong) 20050015. The rats were bred in perversion of day and night with light on at 9 am and off at 9 pm every day. Rats had free access to food and water except during the experiment time. The rats were handled on daily basis.

\subsection{Drugs and Reagents}

Baixiangdan Capsule is composed of White peony root, Cyperus rotundus, moutan. Producted Shandong University of Tranditional Medicine, Batch number: 080201, Acetonitrile (HPLC grade, Merck Company), Formic acid (HPLC grade, U. S. Tedia company), MilliQ ultrapure water, Other reagents were all analytical grade.

\subsection{Bolting Experiment Rats}

Open-field experiment was used to bolt rats of which scores were close to each other. The method of openfield experiment is described below.

\subsection{Method of Confirming the Estrous cycle of the Rats}

The estrum of rats was confirmed by observing the vagina cell shape. The estrous cycle of a rat generally contains four stages: pre-estrous, estrous, meto-estrous and anestrous. The specific procedure was as follows: a small quantity of isotonic $\mathrm{Na}$ chloride was drawn by a dropper, and was dropped repeatedly on a rat's colpo for 2-3 times. The suction fluid was overlaid on glass slide to be naturally air dried. Then it was held by absolute alcohol for 2 to $5 \mathrm{~min}$, followed by being thoroughly accreted by Giemsa stain for 10 to 30 minutes. The cell appearance was observed every time (Table 1). Vaginal smear was conducted 5-9 times every day for 28 days to confirm the estrous cycle of every experimental rat [4].

\subsection{Ethnology Evaluation Method of Estrous Cycle}

Ethnology observation was used to evaluate the estrous
Table 1. Cell morphologic change features of rats' estrous cycle.

\begin{tabular}{|c|c|c|}
\hline Stage & Ovary Changes & $\begin{array}{l}\text { Cell Morphologic Change } \\
\text { Features }\end{array}$ \\
\hline Pre-estrous & $\begin{array}{l}\text { egg chamber } \\
\text { accelerated } \\
\text { growth }\end{array}$ & $\begin{array}{l}\text { All is caryon cellula epithe- } \\
\text { lialis with a small quantity } \\
\text { of keratinocyte }\end{array}$ \\
\hline Estrous & $\begin{array}{l}\text { egg chamber } \\
\text { ovulating }\end{array}$ & $\begin{array}{l}\text { All is acaryotic keratino- } \\
\text { cyte, or several cellula epi- } \\
\text { thelialis }\end{array}$ \\
\hline Metao-estrous & $\begin{array}{l}\text { corepus luteum } \\
\text { forming }\end{array}$ & $\begin{array}{l}\text { blood corpuscle, keratino- } \\
\text { cyte, caryon cellula epithe- } \\
\text { lialis }\end{array}$ \\
\hline Anestrous & $\begin{array}{l}\text { corpus luteum } \\
\text { regression }\end{array}$ & $\begin{array}{l}\text { generous blood corpuscles, } \\
\text { several cellula epithelialis } \\
\text { and cellula mucipara }\end{array}$ \\
\hline
\end{tabular}

cycle. The female rats were put into cages to be observed for their estrous behaviors, such as jumping, crawling, joggling ears, arching back, and the male rat attacking female rat's back (Kow, 1976, Sodersten and Eneroth, 1981). Male rats were not allowed to crawl across female rats' back. Rats showed active estrous behaviors at pre-estrous and estrous (the accepting time), but estrous behaviors decreased or even disappeared at the stages of anestrous and metao-estrous (not accepting time). The interval was two days. Rats which displayed active estrous behaviors were internalized [5].

Thirty rats whose estrous cycles are not in accepting time were selected and weighed. They were randomly divided into three groups: normal control group, PMS liver-qi invasion model group (the invasion model group), PMS liver-qi invasion medication-administeredn group (the invasion medication group). Rats which were not in the accepting time were stimulated continuously for two weeks.

\subsection{PMS Liver-qi Invasion model Method}

The model rats were put into cages which could accommodate interval time and intensity of noise and electricity stimulations freely. Rats were exposed to the noise and electricity stimu-lations once per $5 \mathrm{~min}$ at daytime and once per $10 \mathrm{~min}$ at night. Current flow of $0.5 \mathrm{~mA}$ and voltage $2700-3300 \mathrm{~V}$ were applied. The nervure was $0.3 \mathrm{~s}$ wide. Photographic recording was taken at night and photos were taken once every day [6].

\subsection{Medication}

Medication was orally administered once per day. The administration dosage for rats was $1 \mathrm{ml}$ per $100 \mathrm{~g}$, for five days [7]. Control rats received equal volume of sterile water orally. 


\subsection{Urine Samples}

Rats fed $4 \mathrm{ml}$ sterile water at each day at 9:00 am, and then placed in metabolic cages, using the cylinder collected $2 \mathrm{ml}$ of urine. Urine samples were centrifuged, 20 min with $10000 \mathrm{rpm}$, and the supernatant was taken for further analysis. Following metabolic analysis, rats were tested in the open field.

\subsection{Open-field Experiment Method}

The open-field box was by $100 \mathrm{~cm} 100 \mathrm{~cm} 50 \mathrm{~cm}$. The paries and undersurfaces were black. The undersurface was partitioned into 25 areas with same size by white lines which were called periphery grilles porch. The wall and the rest were called central grilles. The rat's tail was gripped at $1 / 3$ of the root by the runner, and was put into the mesh grille. The rat's behavior changes were record by photographic recording system for three minutes. (1) Crossing score: the score is the number of times that animals cross undersurface grilles. Four claws must all enter the grille. (2) Rearing score: the score is the number of times that animals make a perpendicular act. Four claws must leave empty or hold on to the wall. The open-field experiment score is the total of the crossing score and the rearing score. Simultaneously record the modified time, modified number of times, the number of times staying on the mesh grille, and dejecta number. Each rat was used once after being made into the model, 3 min every time. The differences among groups were compared. The photographic recordings were observed by three staffs, and the concordance was confirmed (Kappa exceeded 0.95) [8].

\subsection{Analysis Conditions}

Chromatographic conditions: Flow $0.4 \mathrm{ml} / \mathrm{min}$, Sample room temperature: $4^{\circ} \mathrm{C}$, Column temperature: $60^{\circ} \mathrm{C}$.

\section{MS conditions:}

Electrospray ion source, Positive ion mode V, 50$1000 \mathrm{Da}$, scan time: $0.1 \mathrm{~s}$, inter time: $0.02 \mathrm{~s}$, Lock mass: $0.01 \mathrm{ml} / \mathrm{min} \operatorname{LE}([\mathrm{M}+\mathrm{H}]+=556.2771)(200 \mathrm{pg} / \mathrm{ml})$, Capillary voltage: $3 \mathrm{kv}$, Cone voltages: $60 \mathrm{v}$, Ion source temperature: $100^{\circ} \mathrm{C}$, Desolvation temperature: $300^{\circ} \mathrm{C}$, Desolvation nitrogen flow: $700 \mathrm{~L} / \mathrm{hr}$, Cone gas flow: 50 $\mathrm{L} / \mathrm{hr}$.

\subsection{Data Processing}

Behavior of experimental data analysis using SPSS 13.0 statistical software, Multiple samples of the comparative analysis of variance with one factor, UPLC-TOF-MS spectra data use the Micromass MarkerLynx software to identify peak and to peak matching, and use principal component analysis to carry out pattern recognition to the control group, model group and administration group.

\section{Experimental Results}

\subsection{Comparison of Open-field Experiment Scores}

In open-field experiments, compared with the normal control group, the crossing scores, rearing scores and open-field experiment total scores of rats in the invasion model group remarkably increased $(\mathrm{P}<0.05)$. The tendency was retrieved remarkably after medication $(\mathrm{P}$ $<0.05)$.

\subsection{The Spectra Results of the urine Metabolic Products}

There was a remarkable difference between control group and model group, but the contrasts were subtle between control group and adminstration group from Figure 1 showed as below.

\subsection{Results of Principal Component Analysis}

The scores chart of the urine metabolic product content through principal component analysis showed as below (Figure 2). There was so close position that we can hardly differentiate the box marks (represent the control group) from the triangle marks (represent the administration group), on the contrary, the diamond marks (represent the model group) distributed far away from other marks.

The box marks represent the control group, the diamond marks represent the model group and the triangle marks represent the administration group.

\subsection{Analysis of Differences in Metabolic Products}

14 potential biological markers had been detected through comparing the metabolic fingerprint difference between model group and control group.

\section{Discussion}

Quantization evaluation of macroscopic appearance of animal samples is very valuable to evaluate liver-related metabolic aspects of the syndrome. Qualitative method and half quantization bound with four-grade score method were adopted to evaluate sample's behaviors and expressions in early works. The specific method was to use four-grade score method (“+”expresses masc. result.) 
to process photographic recording survey results by half quantization, Then, the results were compared with rats in the normal control group. This method just made a corresponding evaluation of model macroscopy appearance by animal clinical symptoms. The modality can't realize the statistical analysis of experimental results. This modality is sufficient as an aiding evaluation method, but it will be too subjective and lacks objective evidence as a major evaluation method for animal macroscopy appearances. In the open-field experiment, compared with the normal control group, the crossing scores, rearing scores and open-field experiment total scores of rats in the invasion model group increased remarkably. Under stimulations, activities and excitement
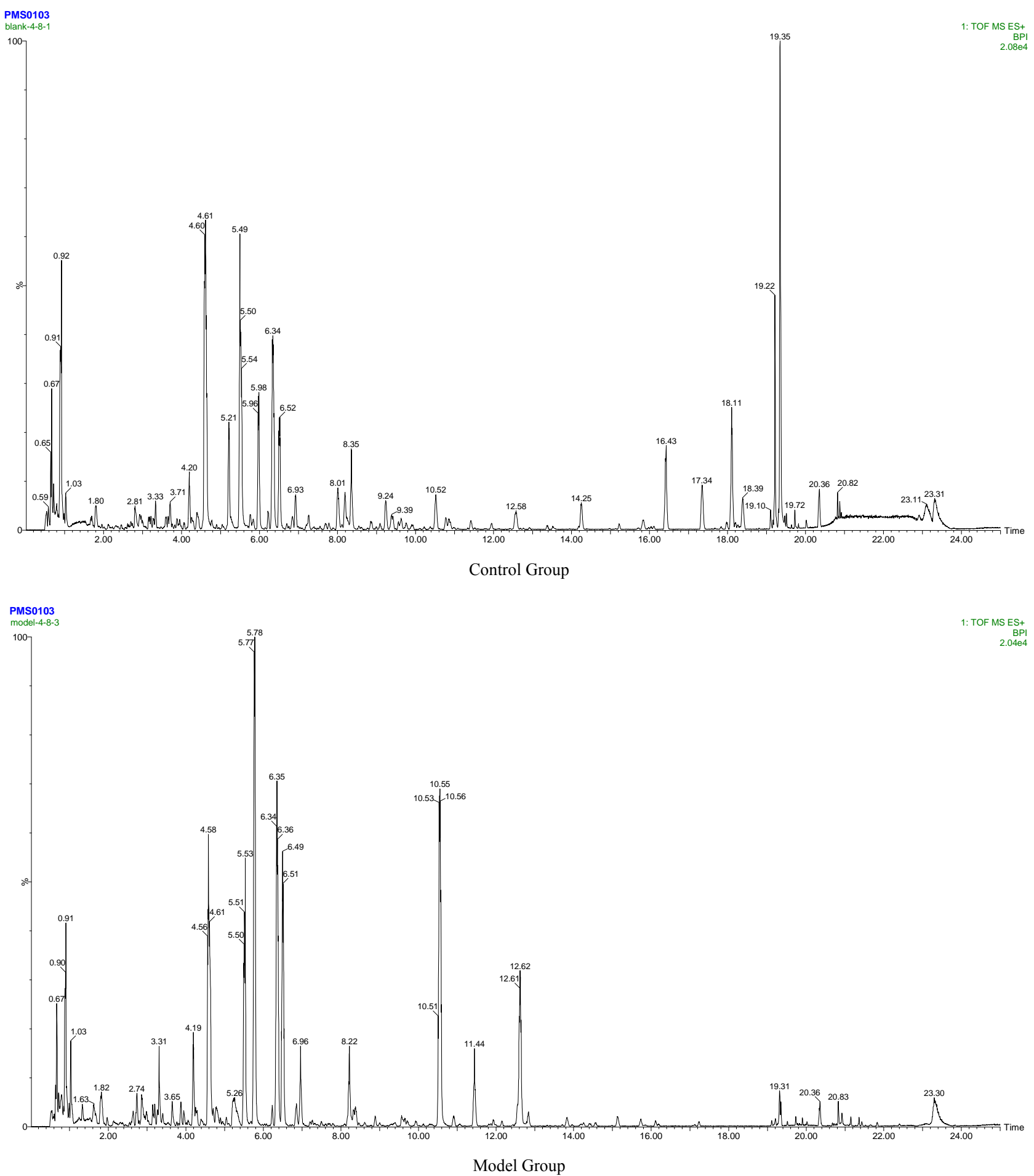


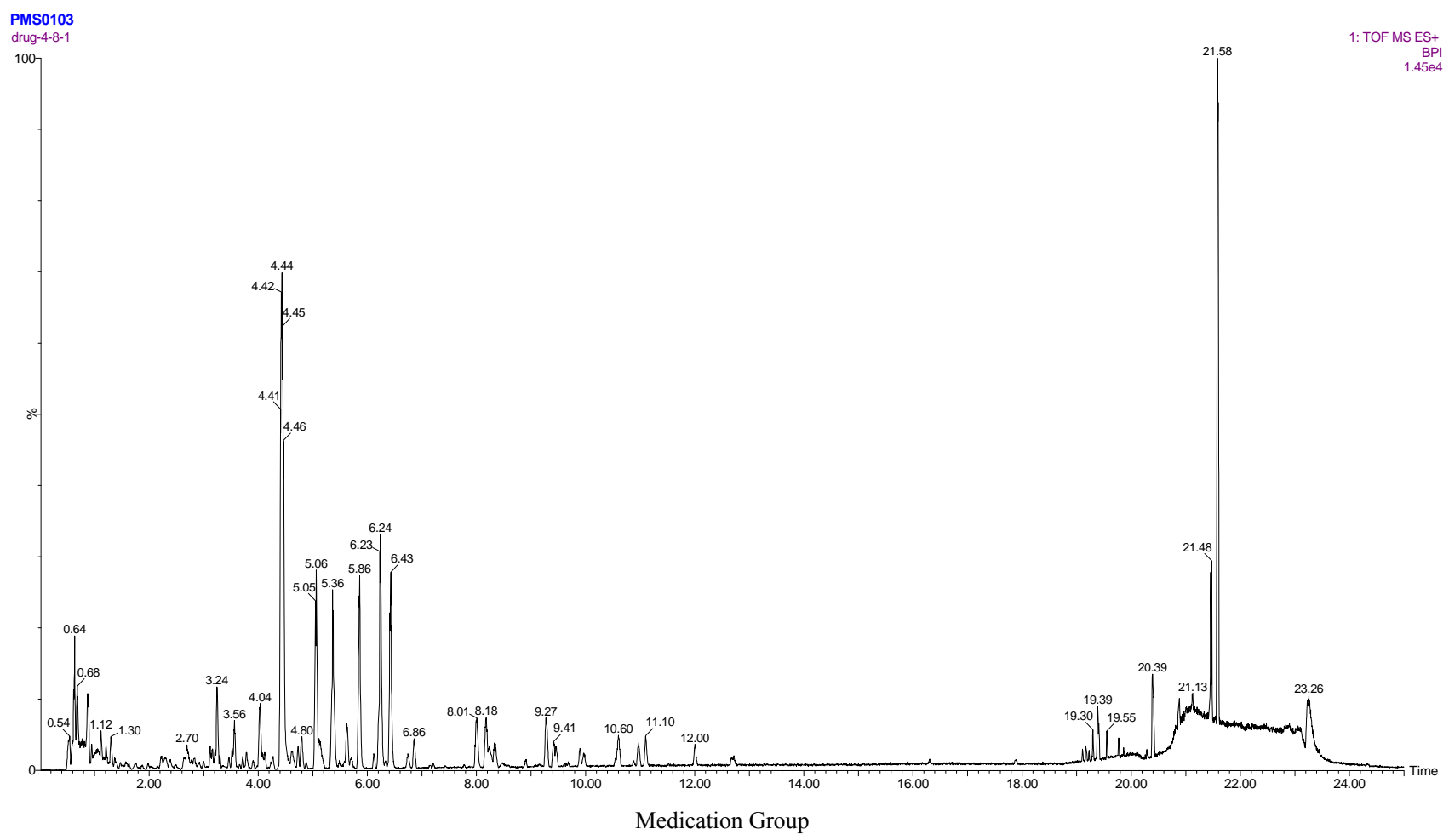

Figure 1. The urine test total ion chromatogram of rats.

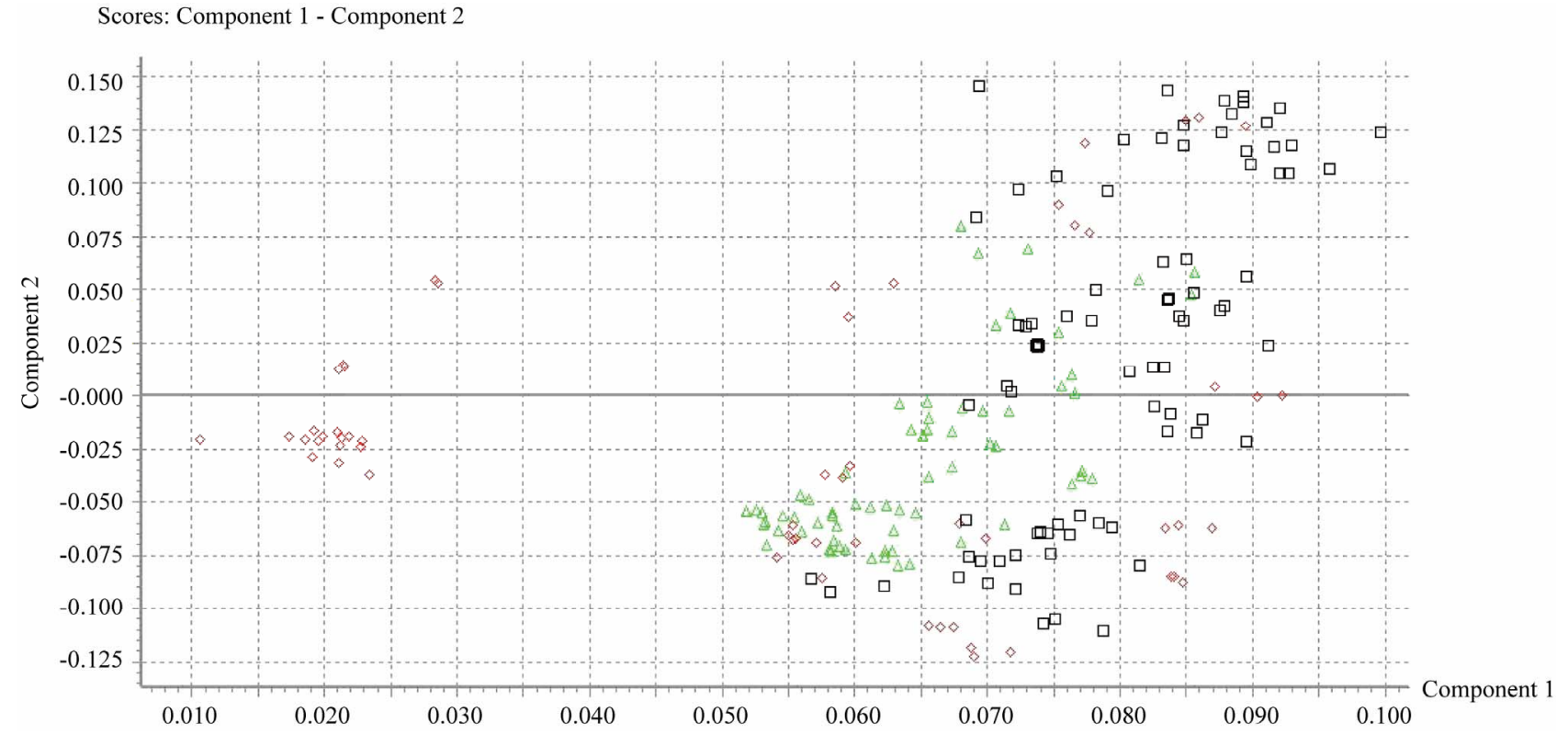

Figure 2. Rat urine metabolic pattern analysis scores chart.

of rats in the invasion model group increased remarkably. After intervention of medication, each group recovered to normal (Table 3). Metabolic fingerprints between the PMS liver-qi invasion model group and the normal control group had also distinguished changes through principal component analysis, which were restored after Baixiangdan Capsules administration (Figure 1 and Figure 2). According to traditional Chinese medicine's theory of symptoms and signs, the model rats displayed proximal clinic symptoms of patients with PMS liver-qi invasion such as anxious emotion, and hyperactivities. Therefore, above-mentioned conclu- 
Table 2. Rat urine UPLC chromatographic conditions.

\begin{tabular}{ccc}
\hline Time $(\mathrm{min})$ & $0.2 \%$ Formic acid (\%) & Acetonitrile (\%) \\
\hline 0 & 100 & 0 \\
18 & 65 & 35 \\
20 & 5 & 95 \\
22 & 5 & 95 \\
25 & 100 & 0 \\
28 & 100 & 0 \\
\hline
\end{tabular}

Table 3. Comparison of open-field experiment scores $(n=10)$.

\begin{tabular}{lccc}
\hline \multicolumn{1}{c}{ Group } & Crossing score & Rearing score & Open-field score \\
\hline $\begin{array}{l}\text { Normal control } \\
\text { group }\end{array}$ & $49.92 \pm 29.11$ & $11.58 \pm 5.41$ & $61.50 \pm 30.93$ \\
$\begin{array}{l}\text { Invasion model } \\
\text { group }\end{array}$ & $64.00 \pm 20.15^{\text {出 }}$ & $15.92 \pm 6.26^{\text {出 }}$ & $79.92 \pm 24.18^{\text {方 }}$ \\
$\begin{array}{l}\text { Medication } \\
\text { group }\end{array}$ & $43.33 \pm 11.11^{\triangle}$ & $9.75 \pm 2.18^{\triangle}$ & $53.08 \pm 12.56^{\triangle}$ \\
\hline
\end{tabular}

There was significant difference when compared with control group $(\mathrm{P}<$ $0.05) ;{ }^{\triangle}$ There was significant difference when compared with model group $(\mathrm{P}<0.05)$

Table 4. Information Sheet potential biological markers.

\begin{tabular}{cclc}
\hline No. & Retention time & \multicolumn{1}{c}{ Identification } & Trends \\
\hline 1 & 1.0 & Methyl guanine & $\uparrow$ \\
2 & 1.1 & 2,3-dihydroxy-3-methyl valerate & $\uparrow$ \\
3 & 4.6 & D-galactosamine & $\downarrow$ \\
4 & 4.7 & 5-Amino acid & $\downarrow$ \\
5 & 5.7 & 2-aminoadipic acid & $\uparrow$ \\
6 & 5.8 & D-Proline & $\uparrow$ \\
7 & 6.3 & Melatonin & $\uparrow$ \\
8 & 6.4 & 3-hydroxy-pyruvate-5-carboxyl & $\uparrow$ \\
9 & 6.5 & 4-hydroxy-glutamic & $\uparrow$ \\
10 & 8.1 & 2,3-dihydro-pyridine acid & $\downarrow$ \\
11 & 8.3 & Deoxy-adenosine & $\downarrow$ \\
12 & 12.6 & 5,7,4 '-genistein & $\uparrow$ \\
13 & 16.3 & Sebacic acid & $\downarrow$ \\
14 & 19.3 & Prostaglandin F2 $\alpha$ & $\downarrow$ \\
\hline
\end{tabular}

sions of qualitative analysis, quantitative judgment and medicine disproof were sufficient to confirm the most important evidence of traditional Chinese medicine's theories of symptoms and signs to the metabolic and behavioral patterns.

This study also found 14 potential biological markers (Table 3) which were relevant with PMS liver-qi invasion symptoms of rats and had not been reported before although some markers thought to be related to the reappraisal regulation processing of emotion such as 2-aminoadipic acid, 4-hydroxy-glutamic. 2-aminoadipic acid was the major metabolic product of lysine metabolism, and the normal metabolism of lysine also depended on the regulation of glutamate. Therefore, 2-aminoadipic acid increasing was associated with glutamate increasing. The relation of 4-hydroxy-glutamic acid and glutamate reflected by the following chemical equation:

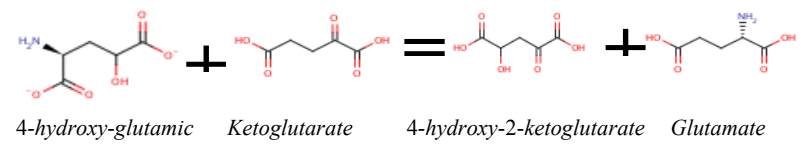

Thus 4-hydroxy-glutamic content growing induced an increase of the glutamate level inferred from above figure. As to the relation between glutamate level and PMS liver-qi invasion symptoms we had done more works. Our previous studies showed that glutamate level was decreased in hypothalamus, while glutamate levels were remarkably increased in cortex and hippocampus comparing PMS liver-qi invasion rats with the control rats [9]. Glutamate is the major excitatory brain neurotransmitter involved in brain protein and glucose metabolism. It can improve the function of the glutamate system neurons [10]. Some researchers had reported that women with premenstrual dysphoric disorder had a higher risk to suffer from emotional disorders. May be glutamatergic system involved in the pathogenesis of affective disorders and it was proved that the drugs which reduce activity of glutamatergic system or inhibit signal transduction of glutamate receptor showed a approximate anti-manic effects [11]. Maybe it can be inferred that glutamate level plays a key role in the course of PMS liver-qi invasion. Other metabolic products mentioned in Table 4 need further exploration about their relation with PMS liver-qi invasion symptomes.

\section{Acknowledgements}

This study was supported by the National Natural Science Foundation of China (No.30973688 and No.30930110), the National Program of Key Basic Research Project (973 Program) (No. 2011CB505102). The authors thank Nashat Abumaria for his excellent manuscript preparation assistance.

\section{References}

[1] J. J. Li, "Clinical Gynecologic Endocrinology and Dysgenesia," Shandong Science and Technology Publishing Company, Jinan, 2003.

[2] T. Schneider and P. Popik, "Increased Depress Ive-Like Traits in All Animal Model of Premenstrual Irritability," Horm behav, Vol. 51, No. 1, January 2007, pp. 142-148.

[3] S. Wei, "Improvement of Premenstrual Syndrome Liver- Qi Invasion, Dpression Animal Model and Study on Micro- 
mechanism of the Liver Failing to Maintain the Normal Flow of Qi," Journal of Shandong University of Traditional Chinese Medicine, Vol. 31, No. 5, May 2007, pp. 1-5.

[4] X. F. Tu, "Effects of Excessive Swimming Exercise Oil the Estrous Cycle of Mice," Chinese Journal of Clinical Rehabilitation, Vol. 36, No. 8, Augest 2004, pp. 8316- 8317.

[5] H.-P. Ho, Marie Olsson and Lars Westberg, "The Serotonin Reuptake Inhibitor Fluoxetine Reduces Sex Steroid-Related Aggression in Female Rats: An Animal Model of Premenstrual Irritability?" Neuropsychopharmacology, Vol. 24, No. 5, May 2001, pp. 502-510.

[6] H. Y. Zhang, M. Q. Qiao and W. C. Zhu, "Variation on Rats' Serum Hormones and Behavior of Premenstrual Syndrome Liver-Qi Invasion," Chinese Traditional Patent Medicine, Vol. 24, No. 2, February 2002, pp. 118-119.

[7] S. Y. Xu, R. L. Bian and X. Chen, "Pharmacological Experiments Methodology," People's Medical Publishing House, Beijing, 2002.
[8] X. W. Liu, H. M. Zhang and H. D. Qu, "Observation and Detection of Behavior on Rat of 'Anger Hurt Qi',' Jiang Su Journal of Traditional Chinese Medicine, Vol. 26, No. 3, March 2005, pp. 53-55.

[9] L. Sun, "PMS Rat Model of Liver against the Permit and Different Brain Regions of Serum Progesterone and Amino Acid Detection Analysis," Master dissertation, Shandong University of Traditional Chinese Medicine, Jinan, 2008.

[10] C. Konradi and S. Heckers, "Molecular Aspects of Glutamate Dysregulation: Implications for Schizophrenia and Its Treatment," Pharmacology \& Therapeutics, Vol. 97, No. 2, February 2003, pp. 153-179.

[11] X. Zhou and X. Q. Wang, "Glutamate and $\Gamma$-Aminobutyric Acid System in Mood Disorder," Chinese Journal of Neuroscience, Vol. 19, No. 2, February 2003, pp. 130-133. 